Conclusion Otherwise stable, well developing former very low birth weight preterm infants are at risk for glucose instability, generally considered as unfavourable. It remains unclear whether this instability is likely to influence long-term outcome and whether continuous feeds are preventive.

\section{SIGNIFICANCE OF HIGH ALKALINE PHOSPHATASE LEVELS WITH IN FIRST TWO WEEKS OF LIFE AMONG EXTREMELY PRE-TERM BABIES}

doi:10.1136/archdischild-2012-302724.1357

AK Gupta, H Ali. Neonatology, The Leeds Teaching Hospitals NHS Trust, Leeds, UK

Background Serum alkaline phosphatase levels (S-ALP) are often high among extremely preterm babies before first two weeks of life. It is not certain whether this represents increased physiological bone turn-over or is a predictor for osteopenia of prematurity.

Aim To study the relationship between osteopenia of prematurity and peak S-ALP levels with in first two weeks among pre-term babies born before twenty-nine weeks gestation.

Methods We evaluated seventy-three extremely pre-term babies born before twenty-nine weeks gestation who were admitted to tertiary neonatal units in Leeds, UK from 01/01/2009 to 31/01/2011. S-ALP, calcium and inorganic phosphate were checked regularly while they were in the neonatal unit. Forty out of seventy three babies had radiographs performed after five weeks post-natal age and were reported by radiologist.

Results In our cohort of seventy-three extremely pre-term babies, $55 \%$ had peak S-ALP levels exceeding $1200 \mathrm{iu} / 1$ (four times the upper limit of normal for adults) with in first two weeks. Infants who developed osteopenia had significantly lower gestational age and birth weight, and were significantly more likely to receive postnatal steroids. Radiologically proven osteopenia developed in $74 \%$ of infants with peak S-ALP exceeding $1200 \mathrm{iu} / 1$ compared to $35 \%$ of infants with peak S-ALP below $1200 \mathrm{iu} / 1$ before two weeks postnatal age ( $p$-value 0.014).

Conclusion S-ALP exceeding $1200 \mathrm{iu} / \mathrm{l}$ with in two weeks postnatal age is associated with 2.1 fold increased risk of development of osteopenia in extremely pre-term infants.

\section{EFFECT OF MODE OF DELIVERY ON MORTALITY AND MORBIDITY IN VERY LOW BIRTH WEIGHT NEONATES WITH RESPIRATORY DISTRESS SYNDROME}

doi:10.1136/archdischild-2012-302724.1358

A Dursun, BS Karagol, N Hakan, N Karadag, A Zenciroglu, N Okumus, D Dilli, S Beken. Neonatology, Dr Sami Ulus Maternity and Children's Health and Diseases Training and Research Hospital, Ankara, Turkey

Background \& Aim: It is known that wet lung syndrome and pulmonary maladaptation are more frequent in infants delivered by cesarean section while the effect of mode of delivery on RDS is unknown. In this study, we analyzed the effect of the mode of delivery on RDS outcome in very low birth weight (VLBW) neonates.

Methods Data of all the VLBW neonates with respiratory distress syndrome (RDS) between 2007 and 2012 was retrospectively analyzed. Gestational age, gender, birth weight, mode of delivery, necrotizing enterocolitis (NEC), intracranial bleeding (ICH), patent ductus arteriosus (PDA), retinopaty of prematurity (ROP), broncopulmonary dysplasia (BPD) were noted.

Results A total of 186 newborns were diagnosed as VLBW neonates with RDS among the 5980 neonates hospitalized in NICU. Mean birth weight and gestational age were $1058 \pm 261$ grams and $28 \pm 2.7$ weeks, respectively. Cesarean delivery rate was $62.7 \%$. There were no differences with respect to birth weight, gestational age and gender between mode of delivery. There was no significant relationship between the NEC, PDA, ROP, BPD and mode of delivery. Also no significant relationship between the mode of delivery and mortality was determined. On the other hand, ICH was significantly higher in neonates delivered vaginally ( $48 \%$ vs. $31 \%$, p $<0.05$ ).

Conclusion Mode of delivery has no effect on the mortality and morbidity of RDS in VLBW neonates but ICH was significantly higher in normal vaginal delivery group. Therefore, mode of delivery should be decided on the basis of obstetrical indications.

\section{NEONATAL MORTALITY AND MORBIDITY IN DIFFERENT SOCIOECONOMIC CLASSES IN SOUTHWEST OF IRAN}

doi:10.1136/archdischild-2012-302724.1359

N Maharlouei, KB Lankarani. Health Policy Research Center, School of Medicine, Shiraz University of Medical Sciences, Shiraz, Iran

Background and Aims Socioeconomic inequality in infant mortality and morbidity are challenging subjects even in many developed countries. In this study we compared neonatal mortality and morbidity in different socioeconomic status (SES) in Fars, Iran.

Methods A cross-sectional study was conducted in Fars, the fifth populated province in Iran from March to October, 2011. Using cluster random sampling method, data was collected by interviewing mothers two months after delivery and filling the check list from their health file. We categorized interviewees into low, middle and high SES according to their education, job, and wealth.

Results 2106 (93.6\%) mothers participate in this study. Of them $11(0.9 \%)$ lost their fetus in pregnancy, 8 mothers $(0.4 \%)$ experienced still birth while 18 mothers (0.8\%) lost their baby in neonate period. $97.3 \%$ of mothers gave birth in the hospital not related to their socioeconomic ranks $(\mathrm{P}=0.1)$. Also, no association was found between SES and APGAR ( $\mathrm{P}=0.06)$, frequency of fetal and neonatal dead $(p=0.1)$, and admission in neonatal intensive care units $(\mathrm{P}=0.2)$. Additionally, frequency of birth trauma (fracture of humorous, clavicle, femur and skull) did not statistically differ in these groups. However, congenital anomaly $(\mathrm{P}=0.005)$, icterus $(\mathrm{P}=0.004)$, neonatal convulsion $(\mathrm{P}=0.003)$ and neonatal infection $(\mathrm{P}=0.007)$ were highest in middle socioeconomic and lowest in wealthy group. Conclusions This study showed good access to health facilities irrespective of SES. More attention should be paid to neonates of middle SES group, since they suffered the most from neonatal morbidity.

\section{MECONIUM/STOOL AND URINARY PATTERNS OF HEALTHY TURKISH NEWBORNS}

doi:10.1136/archdischild-2012-302724.1360

${ }^{1} S M$ Kayıran, ${ }^{2} E$ Eroğlu, ${ }^{3} \mathrm{PG}$ Kayıran, ${ }^{4} \mathrm{~S}$ Sazak, ${ }^{5} \mathrm{~B}$ Gurakan. 'Deapartment of Pediatrics; ${ }^{2}$ Department of Pediatric Surgery; ${ }^{3}$ Department of Pediatrics, American Hospital; " ${ }^{4}$ Deapartment of Pediatrics, Ministry of Health, Okmeydani Training Hospital; ${ }^{5}$ Department of Pediatrics, Division of Neonatology, American Hospital, Istanbul, Turkey

Background and Aims Routine follow-ups of healthy newborns for up to $48 \mathrm{~h}$ for those delivered by normal vaginal delivery (NVD) and for up to $96 \mathrm{~h}$ for those delivered by cesarean section (CS) are highly recommended as they facilitate the early detection of certain problems.

Objectives To investigate the meconium/stool and urinary patterns of healthy Turkish neonates, and to determine whether they correlate with delivery mode, birth weight and feeding method and frequency.

Methods Newborns with a gestational age of $\geq 34$ wk were included. The frequency of meconium/stool and urine passage and the delivery mode, birth weight and feeding method and frequency were recorded throughout their hospital stay. 